\title{
Collision-Free Path Planning for Automated Vehicles Risk Assessment via Predictive Occupancy Map
}

\author{
Dan Shen, Yaobin Chen, Lingxi Li, Stanley Chien \\ Transportation Active Safety Institute \\ Indiana University-Purdue University Indianapolis, USA
}

\begin{abstract}
Vehicle collision avoidance system (CAS) is a control system that can guide the vehicle into a collision-free safe region in the presence of other objects on road. Common CAS functions, such as forwardcollision warning and automatic emergency braking, have recently been developed and equipped on production vehicles. However, these CASs focus on mitigating or avoiding potential crashes with the preceding cars and objects. They are not effective for crash scenarios with vehicles from the rear-end or lateral directions. This paper proposes a novel collision avoidance system that will provide the vehicle with allaround (360-degree) collision avoidance capability. A risk evaluation model is developed to calculate potential risk levels by considering surrounding vehicles (according to their relative positions, velocities, and accelerations) and using a predictive occupancy map (POM). By using the POM, the safest path with the minimum risk values is chosen from 12 accelerationbased trajectory directions. The global optimal trajectory is then planned using the optimal rapidly exploring random tree $\left(\mathrm{RRT}^{*}\right)$ algorithm. The planned vehicle motion profile is generated as the reference for future control. Simulation results show that the developed POM-based CAS demonstrates effective operations to mitigate the potential crashes in both lateral and rear-end crash scenarios.
\end{abstract}

\section{INTRODUCTION}

Autonomous vehicles (AVs) have become a popular research area in both the automotive industry and academia with the objective of minimizing risks and enhancing safety and comfort. Based on the National Highway Traffic Safety Administration (NHTSA), smashing into the rear of the car ahead is the top cause of vehicle accidents, contributing up around $30 \%$ of all traffic accidents annually [1]. Collision avoidance system (CAS) is one of vehicle active safety technologies for dealing with both lane-departure and forward-collision problems, which has been designed and implemented on some production vehicles. D. Sam [2] concluded that most road accidents occured due to human error, and over $90 \%$ of those accidents were caused by visual information acquisition problems. However, most of the currently developed CASs were designed to mitigate crashes based on the errors caused by the ego vehicle (e.g., driver distraction and drowsiness [3]) and static objects on road, such as lane markings, road edges, and parked vehicles. These systems are not effective when dealing with crashes caused by other vehicles at fault. On the other hand, except for the lane/road departure cases, traditional CAS mainly focuses on handling the potential crashes from the ego vehicle to the preceding cars. These systems do not work at all for rear-end or lateral crash scenarios. For aforementioned crash scenarios, if human drivers have been operating the vehicle, the accidents can be handled since drivers would be able to make the defensive maneuvers. Unfortunately, AVs cannot deal with such crash situations to mitigate or alleviate the collisions initiated by other moving vehicles [4]. Many technical methods have been proposed in this area to improve the intelligence of AVs for risk evaluation and motion planning in simple scenarios, e.g., only considering two vehicles on the two-lane road [5]. In this work, a collision mitigation system with features of all-around (360-degree) collision avoidance capability is proposed. The objective is to evaluate the surrounding risks of the ego vehicle based on the driving environment, and then find a feasible and smooth path from the start point to the endpoint without colliding with any obstacles.

The risk assessment module is a prerequisite for motion planning. Risk assessment computes the danger of potential paths of the ego vehicle based on the surrounding environment $[6,7]$. Current risk assessment methods are not designed for multi-vehicle collision scenarios, and the potential risks from other vehicles and the surrounding environment are modeled separately. To overcome this shortfall, a multi-vehicle risk assessment needs to be developed for advanced CAS. Based on the results from risk assessment module, the motion planning module finds the safest trajectory that the ego vehicle should follow over the next short period. However, the ego vehicle may collide with other vehicles or obstacles on the road when mitigating the potential collison with a target vehicle, which indicates that all risks of the vehicle surrounding environment need to be considered. In order to address this issue, several researchers have modeled the risks of cars using the potential field method [8]. The multi-vehicle scenarios may have been modeled and represented, but the risks from surrounding vehicles were obtained according to the current positions. If the surrounding vehicle speed is high, the collision avoidance system camnot rely on the planned collision-free path. Therefore, a prediction for trajectories of surrounding vehicles is necessary. Meanwhile, the control techniques will be utilized to enforce the ego vehicle to follow the desired vehicle motion profiles [9-11]. 
The presented work in this paper was primarily motivated by [12], where the authors applied the predictive occupancy map as a motion planning approach for avoiding crashes on road. There are three limitaions for the proposed method in [12]: (1) the two-dimensional crash danger assessment of surrounding vehicles covers only about $37.5 \%$ of surrounding area around the ego vehicle , (2) the planned acceleration profile does not include the reference sequence of yaw angle and velocities on the desired trajectory, (3) the collision-free trajectory generated in [12] is not global. In this paper, we propose to generate the collision-free trajectory in the global sense by utilizing the optimal rapidly exploring random tree (RRT*). The main contributions of the paper are summarized as follows:

1) The dynamic traffic condition on the straight road was modeled using POM, which is able to identify risk levels for all surrounding environments in both the spatial and temporal spaces.

2) The ego veihcle motion profile is planned based on the multi-vehicle risk assessment through POM, which includes reference sequence of positions, velocity, acceleration, and yaw angle.

3) The collision-free trajectory in the global sense is obtained by utilizing the optimal rapidly exploring random tree $\left(\mathrm{RRT}^{*}\right)$ with $\mathrm{POM}$.

The remainder of this paper is organized as follows. The overall collision avoidance system is discussed in Section ПI. A brief overview of the POM is introduced in Section III. Section IV explains the motion planning and trajectory generation for collision mitigation. Simulation results of one lateral collision scenario are illustrated in Section V. Finally, the results and conclusions are presented in Section VI.

\section{THE OVERALl COLLISION AVOIDANCE SYSTEM}

This section mainly introduces the basic concept and operation process of the proposed collision avoidance system. The execution conditions and transition of the driving modes of the automated vehicle under control is discussed based on the risk level of the ego car. The overall architecture for the proposed CAS control is also described.

\section{A. The Basic Concept and Operation Process of MDCAS}

This paper proposes a multi-directional collision avoidance system (MDCAS), which can evaluate the potential risks associated with surrounding vehicles from all directions and can generate the corresponding collision-free trajectory. As an operating mode of automated vehicles, original CAS was desioned to eliminate and/or minimize human errors from the ego vehicle. The proposed collision mitigation system obtains the risk values from the perception information in the sensing system for all surrounding objects and applies the correct maneuvers accordingly. The overall structure and proposed decisionmaking process of the CAS are given in Fig. 1. We assume that the vehicle can continuously monitor the surrounding environment through various sensors and evaluate the risk levels of the ego vehicle. If the risk value of the ego car $\left(\right.$ Risk $\left._{e, g o}\right)$ is greater than the pre-determined risk threshold (Risk threshold $)$, the proposed collision avoidance system will be activated which applies the motion planning algorithm to calculate the future safest tracking trajectory. At the same time, human driving operation or any automated operation, such as lane keeping or mode, will be overridden. Once the CAS is activated, it will maintain the active status for a certain period of time. The CAS total activation time of MDCAS, $T_{M D C A S}$, is explained below [12]:

$$
T_{M D C A S}=m \times \Delta t\left(T_{0} \leq T_{M D C A S} \leq T_{f}\right)
$$

where $T_{0}$ is the start time of the proposed CAS, $T_{p}$ is the final time of the execution of the CAS, $m$ is the iteration number of the algorithm, and $\Delta t$ is the sampling period of the system.

As shown in Fig. 1, the spatial and temporal traffic conditions are modeled using a predictive occupancy map, and then the risk assessment module computes the risks of

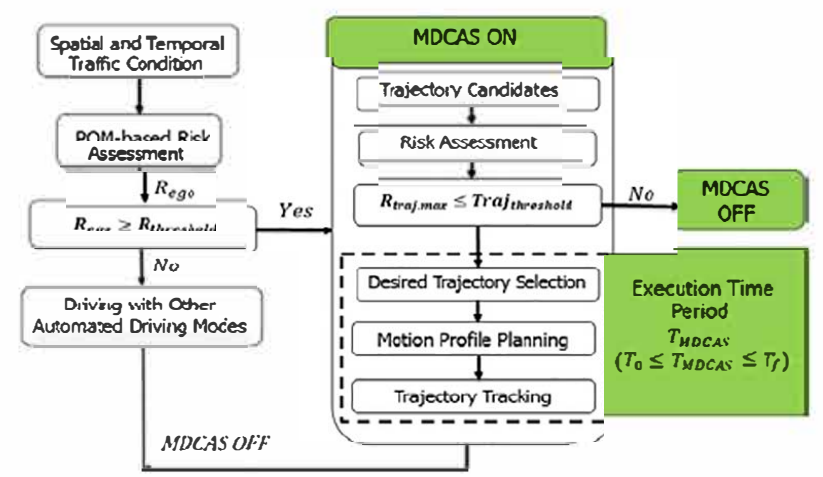

Fig. 1. Flowchart of multi-directional collision avoidance system.

the ego vehicle. The CAS is ON when Risk $k_{\text {ego }} \geq$ Risk $k_{\text {threshold }}$. Otherwise, the AV will operate in other automated driving modes, such as lane following control mode or automatic lane change mode. Note that when the CAS is ON, it does not mean that the maneuvers from CAS are executed but it implies that the sensing system is continuously monitoring the surrounding environment and evaluating the risks of the ego vehicle. If the CAS is ON and the evaluated risk values of all trajectory candidates are higher than the trajectory threshold value ( $\left.T r a j_{\text {threshold }}\right)$, it means that current driving area is safer than any surrounding driving areas. Therefore, the ego car should maintain the previously determined operation and wait for an appropriate evasion space for collision avoidance..

\section{OVERVIEW OF THE PREDICTIVE OCCUPANCY MAP}

Collision risks considered in this paper are related to two types of factors. One is the static and moving vehicles surrounding the ego vehicle. The other is related to the surrounding environment that include the drivable areas and traffic lane markings on road. 
Predictive occupancy map (POM) is defined as the top view of an area surrounding the ego vehicle (e.g., 8 car width and 8 car length). A POM is devided into a finite set of squares called cells (e.g., a cell is $0.25 \mathrm{~m} * 0.25 \mathrm{~m}$ ). Each cell in the occupancy grid has a value representing the probability of the occupancy of that cell by an object. The probability value close to 1 means that there is a high probability that the cell contains an obstacle, and the value close to 0 means that there is a high probability that the cell is not occupied by any object. The ego vehicle coordinate system will be used in the POM modeling process. In the ego vehicle coordinate system, the ego vehicle is always viewed as the origin with respect to other surrounding environments. The vehicle is assumed to move in the $\mathrm{x}$ direction of the ego coordinate systems.

The POM can predict future vehicle positions based on the extrapolation of the relative position, velocity, and acceleration of the neighboring vehicles to the ego vehicle in the ego vehicle coordinate system. The level of the risk in a cell is assigned risk scores from 0 and 10 , with 0 being no risk and 10 being the highest risk. In order to accomplish the risk assessment and successful motion planning, the ego vehicle dynamic obtained via the sensing system is necessary, which can be represented as follows:

$$
V e h_{\text {ego }}=\left[P_{\text {ego }, x}, P_{\text {ego }, y}, V_{\text {ego }, x}, V_{\text {ego }, y}, A_{\text {ego }, x}, A_{\text {ego }, x}\right]
$$

where $V e h_{e g o}$ is the vector of the ego vehicle dynamic information. $P_{e g o, x}$ is the x coordinate of the ego vehicle position, $P_{e g o, y}$ is the y coordinate of the ego vehicle position, $V_{e g o, x}$ is the ego vehicle velocity along the x-axis, $V_{e g o, y}$ is the ego vehicle velocity along the y-axis, $A_{e g o, x}$ is the ego vehicle acceleration along the x-axis, and $A_{e g o, y}$ is the ego vehicle acceleration along $y$-axis, in the world coordinate systems. The surrounding vehicle $\mathrm{n}$ in the world coordinate systems can be represented as follows:

$$
\operatorname{Veh}_{n}=\left[P_{n, x}, P_{n, y}, V_{n, x}, V_{n, y}, A_{n, x}, A_{n, y}\right]
$$

If we consider only the relative motion of all vehicles to the ego vehicle in the ego vehicle coordinate systems, the values of ego vehicle positions, velocities, and accelerations are all 0 in the ego vehicle coordinate systems. Fig. 2 shows the dynamic information of surrounding vehicles relative to ego vehicle, which can be expressed as (4):

$$
\begin{aligned}
& V e h_{r n}=V e h_{n}-V e h_{e g o} \\
= & {\left[P_{r n, x}, P_{r n, y}, V_{r n, x}, V_{r n, y}, A_{r n, x}, A_{r n, y}\right] }
\end{aligned}
$$

where $V e h_{n}$ is the dynamic information vector of the surrounding vehicle $\mathrm{n}$ ( $\mathrm{n}$ is the obstacle index) in the ego vehicle coordinate system. $P_{n}$ is the surrounding vehicle position, $V_{n}$ is the surrounding vehicle velocity, $A_{n}$ is the surrounding vehicle acceleration. $V e h_{r n}$ is the relative dynamic information vector of surrounding vehicle $\mathrm{n} . P_{r n}$ is the relative position of neighboring vehicle $\mathrm{n}, V_{r n}$ is the relative velocity of neighboring vehicle $\mathrm{n}, A_{r n}$ is the relative acceleration of neighboring vehicle $\mathrm{n}$, and subscripts $\mathrm{x}$ and $\mathrm{y}$ in (3) and (4) represent the longitudinal and lateral directions in the ego vehicle coordinate system, respectively. The ego vehicle coordinate system will be used in the rest of the paper.

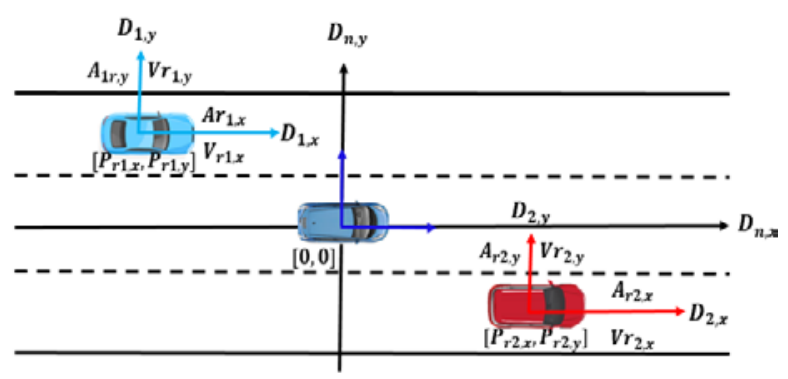

Fig. 2. Surrounding vehicle dynamic information based on the ego vehicle coordinate system.

\section{A. Costmap Generation for Surrounding Vehicles}

The costmap is also called an occupancy map that holds information about the environment, such as the surrounding areas that the ego vehicle cannot traverse. The costmap is a component of POM, in which each cell is associated with a cost value in the range $[0,1]$ representing the cost of navigating through that cell. A cost value is an estimation of the future driving risk of the ego vehicle with respect ot the surrounding vehicles and obstacles.

The proposed collision avoidance system is designed for avoiding the potential imminent crash within a short time period. Thus, we use the constant acceleration (CA) vehicle model to predict vehicle future movingtrajectories. The equation of motion for the CA model in the ego vehicle coordinate systems is

$$
\mathrm{P}=\frac{1}{2} A_{r} \cdot t^{2}+V_{r} \cdot t
$$

where $\mathrm{P}$ is the driving distance within time interval $\mathrm{t}$.

According to [12], the advanced time-to-occupancy (ATTO) is defined as

$$
\operatorname{ATTO}_{n}\left(D_{n}\right)=\frac{D_{n}}{V_{r n}+d \cdot A_{r n}}
$$

where $D_{n}$ is the position of the surrounding vehicle $n$ after traveling time $\mathrm{t}$, and subscripts $\mathrm{x}$ and $\mathrm{y}$ represent the longitudinal and lateral directions, respectively. The time for applying the vehicle relative acceleration is $\mathrm{d}$. The larger the $\mathrm{d}$ value, the longer time using acceleration term.

Since the risk of vehicles has not been specifically designated in the previous literature, in this paper, the definition given in [12] will be used to represent the risk of vehicles considering the relationship between positions and traveling time. The POM is defined as the reciprocal of the previously defined term ATTO at a certain driving space, which can be represented below.

$$
R_{n}\left(D_{n}\right)=\operatorname{ATTO}_{n}^{-1}\left(D_{n}\right)=\frac{V_{r n}+d \cdot A_{r n}}{D_{n}}
$$

When ATTO is 0 , which implies that $D_{n}=0$ and vehicle crashes, $R_{n}\left(D_{n}\right)$ becomes infinity. Thus, we set the maximum risk value $R_{\max }=10 \quad(\mathrm{ATTO}=0.1 \mathrm{sec})$ 
based on the system sampling time $0.1 \mathrm{sec}$, which is the shortest time interval the vehicle can avoid crash. In this case, the acceleration gain $d$ is set as 0.1 . Therefore, the comprehensive risk representations for a two-dimensional driving region based on the center of surrounding vehicles can be concluded considering the vehicle dimension by Equations (8) - (11), which is illustrated in Figure 3.

Case 1: when $\left(\operatorname{abs}\left(D_{n, y}-P_{r n, y}\right)<0.5 \mathrm{~W}\right)$

$R_{n}\left(D_{n, x}, D_{n, y}\right)=\frac{a b s\left(V_{r, x}+d \cdot A_{r, x}\right)}{a b s\left(D_{n, x}-P_{r n, x}\right)-0.5 L}$,

Case 2: when $\left(\operatorname{abs}\left(D_{n, x}-P_{r n, x}\right)<0.5 \mathrm{~L}\right)$

$\operatorname{Risk}_{n}\left(D_{n, x}, D_{n, y}\right)=\frac{a b s\left(V_{r, y}+d \cdot A_{r, y}\right)}{a b s\left(D_{n, y}-P_{r n, y}\right)-0.5 W}$,

Case 3: when $\left(\operatorname{abs}\left(D_{n, y}-P_{r n, y}\right) \leq\right.$ $\left.0.5 \mathrm{~W} \& \operatorname{abs}\left(D_{n, x}-P_{r n, x}\right) \leq 0.5 \mathrm{~L}\right)$

$$
\operatorname{Risk}_{n}\left(D_{n, x}, D_{n, y}\right)=R_{\text {max }}
$$

Case 4: when $\left(\operatorname{abs}\left(D_{n, y}-P_{r n, y}\right)>\right.$ $\left.0.5 \mathrm{~W} \& \operatorname{abs}\left(D_{n, x}-P_{r n, x}\right)>0.5 \mathrm{~L}\right)$

$$
\begin{aligned}
& \operatorname{Risk}_{n}\left(D_{n, x}, D_{n, y}\right)=\left(\frac{a b s\left(D_{n, x}-P_{r n, x}\right)-0.5 L}{a b s\left(V_{r, x}+d \cdot A_{r, x}\right)}+\right. \\
& \left.\frac{a b s\left(D_{n, y}-P_{r n, y}\right)-0.5 W}{a b s\left(V_{r, y}+d \cdot A_{r, y}\right)}\right)^{-1}
\end{aligned}
$$

where $\mathrm{L}$ and $\mathrm{W}$ are the vehicle length and width, respectively, and subscripts of $\mathrm{x}$ and $\mathrm{y}$ represent the $\mathrm{x}$-axis and the y-axis, respectively.

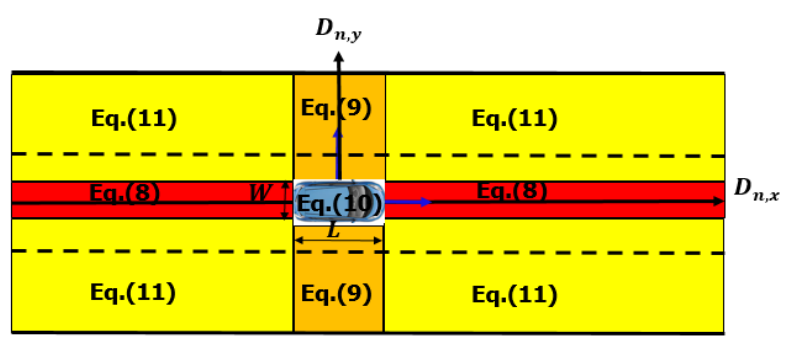

Fig. 3. Planar collision risk evaluation of surrounding vehicles based on ego vehicle local coordinate system.

Based on the equations (8)-(11), we can obtain the risk values of every surrounding vehicle that can be detected by the perception system around the ego vehicle. The integration of all risks should be calculated relative to the ego vehicle. Thus, the overall risk level $R_{\text {overall }}$ is determined by taking the maximum risk values at every driving region.

$$
R_{\text {overall }}=\max \left(R_{1}, R_{2}, \ldots, R_{n}\right)
$$

\section{B. Costmap Generation for Surrounding Environments}

The driving environment considered in this paper consists of two types: drivable traffic area and lane markings. For the drivable traffic area, we need to make sure that the ego vehicle will operate in the road area without colliding with roadside objects such as metal guardrails or concrete dividers. For detected lane markings, risk of the ego vehicle from lane change will be considered, which means the lane change maneuver is not preferred if other maneuvers can successfully mitigate crashes.

Risks regarding the drivable regions are calculated based on vertical road edges on the road such as curb or concrete divider. Areas inaccessible to the ego vehicles will be set as the maximum risk value of 10 . As can be seen in Fig. 4, the formula of drivable boundaries is presented below.

$$
\begin{gathered}
R_{\text {drivable }}=R_{\text {max }}\left(\operatorname{step}\left(D_{n, y}-P_{r n, y}-\text { Bound }_{\text {left }}\right)+\right. \\
\left.\operatorname{step}\left(-\left(D_{n, y}-P_{r n, y}\right)-\text { Bound }_{\text {right }}\right)\right)
\end{gathered}
$$

where Bound $_{\text {left }}$ and Bound right $_{\text {are the distances }}$ from left and right road boundaries to the center of the ego vehicle, respectively, and step represents the step function.



Fig.4. Drivable region risk.

Crossing lanes on highways can increase the collision risk of the ego vehicle with surrounding vehicles. In addition, in some specific scenarios, the ego vehicle may not have enough space to apply emergency lane change. Thus, if the risks of lane change or driving in a longitudinal direction are similar, the longitudinal deceleration to avoid crash will be selected. Therefore, the risks of a traffic lane can be written as

$R_{\text {lane }}=-\left|R_{\text {lane-max }} \cdot \cos \left(\frac{\pi\left(D_{n, y}-P_{r n, y}\right)}{W_{\text {lane }}}\right)\right|+R_{\text {lane-max }}$

where $W_{\text {lane }}$ is the width of lane marking, and $R_{\text {lane-max }}$ is the maximum risk value of lane markings. It can be seen from the equation above, the closer to the lane marking, the greater the risk value on the costmap.

Since we have obtained both risks of drivable area and lane, the total risk of the surrounding environment is

$$
R_{\text {environment }}=\max \left(R_{\text {drivable }}, R_{\text {lane }}\right)
$$

Therefore, the predictive occupancy map is combined with all risks described above, such as surrounding vehicle risks and environmental risks. The POM of the ego vehicle at the origin is represented as:

$$
\mathrm{POM}=\max \left(R_{\text {overall }}, R_{\text {environment }}\right)
$$

\section{MOTION PLANNING AND TRAJECTORY GENERATION}

This section will mainly describe how the safest acceleration-based route is selected through POM, and how 
to obtain the vehicle moving profile in the global sense after getting the desired trajectory via RRT* algorithm.

\section{A. Risk Assessment of Trajectory Candidates}

Twelve pre-defined acceleration-based trajectories which are 30 degrees apart from each other on POM according to [12] are determined and can be shown below.

$\operatorname{Traj}_{i}=\sqrt{P_{i x-\text { lim }^{2}}{ }^{2}+P_{i y-l i m}}{ }^{2}$ for $\mathrm{i} \in \mathrm{T}[1,2, \ldots, 12]$

where $P_{i x-l i m}$ and $P_{i y-l i m}$ are the limited reachable longitudinal position and lateral position, respectively. Since the ego vehicle local coordinate is used, $P_{i x-l i m}$ can be computed by following the general CA prediction model with the initial zero speed. Regarding the limited reachable lateral position, we assume that vehicle lateral speed is zero when arriving the desired position (a distance of changing one lane width) for collision avoidance, which means the vehicle will accelerate during the first half active time of CAS, and decelerate during the second half active time of CAS. The CAS final time $\left(t_{f}\right)$ and the reachable limits can be represented as follow:

$$
\begin{aligned}
& P_{i x-\lim }=\frac{1}{2} A_{\text {ix-lim }} \cdot \cos \left(\frac{\pi(\mathrm{i}-1)}{6}\right) \cdot t_{f}^{2} \\
& P_{\text {iy-lim }}=\frac{1}{4} A_{\text {iy-lim }} \cdot \sin \left(\frac{\pi(\mathrm{i}-1)}{6}\right) \cdot t_{f}^{2} \\
& t_{f}=\sqrt{4 \cdot W_{\text {lane }} \cdot \frac{1}{\mu g}}
\end{aligned}
$$

where $A_{i x-l i m}$ and $A_{i y-l i m}$ are the acceleration limits along the $\mathrm{x}$-axis and $\mathrm{y}$-axis of the ego vehicle, which will ensure vehicle stability while CAS activating [13]. $\mu$ is the road surface friction coefficient, and $\mathrm{g}$ is the gravity constant.

Among the pre-determined 12 trajectory options, it is significant to find the safest trajectory through the comparison process. 10 waypoints on each of the 12 trajectory candidates are captured evenly along the path since every length of the trajectory may be different, and their max, mean, and min values are computed as [12]:

$$
\begin{array}{r}
R_{\text {traji-max }}=\max \left\{\sum_{k}^{10} \operatorname{POM}\left(\frac{k}{10} \cdot P_{i x}, \frac{k}{10} \cdot P_{i y}\right)\right\} \\
R_{\text {traji-min }}=\min \left\{\sum_{k}^{10} \operatorname{POM}\left(\frac{k}{10} \cdot P_{i x}, \frac{k}{10} \cdot P_{i y}\right)\right\} \\
R_{\text {traji-mean }}=\operatorname{mean}\left\{\sum_{k}^{10} \operatorname{POM}\left(\frac{k}{10} \cdot P_{i x}, \frac{k}{10} \cdot P_{i y}\right)\right\}
\end{array}
$$

To compare the assigned 12 trajectories, the following process is followed. First of all, the maximum value of the desired trajectory cannot be over the threshold value $\left(\right.$ Traj $\left._{\text {threshold }}=4\right)$. The value of 4 is determined based on the simulation results, which makes sure that there are appropriate number of candidate trajectories satisfied.

$$
R_{\text {traji-max }} \leq \text { Traj }_{\text {threshold }}
$$

After meeting the requirement of the first condition, the trajectory with the lowest mean is chosen.

$$
\operatorname{Traj}_{\text {desired }}=\min _{i \in T}\left(R_{\text {traji-mean }}\right)
$$

If more than one trajectory satisfies the second condition given in (25), the trajectory with the lowest min value will be chosen, and this is the final chosen safest accelerationbased trajectory for the vehicle on the predictive occupancy map.

$$
\operatorname{Traj}_{\text {desired }}=\min _{i \in T}\left(R_{\text {traji-min }}\right)
$$

\section{B. Trajectory Generation}

The future driving direction and vehicle acceleration can be obtained from the best final acceleration-based trajectory depend on the POM in the previous section. Thus, the final collision avoidance position $\left(P_{x}, P_{y}\right)$ at time $t_{f}$ based on the global coordinate system is computed as

$$
\begin{aligned}
& P_{x}=P_{x o}+V_{x o} \cdot t_{f}+\frac{1}{2} A_{i x-\lim } \cdot \cos \left(\frac{\pi(\mathrm{i}-1)}{6}\right) \cdot t_{f}^{2} \\
& P_{y}= \begin{cases}P_{y o}+W_{\text {lane }}, & (\text { left lane change }) \\
P_{y o}-W_{\text {lane }}, & (\text { right lane change })\end{cases}
\end{aligned}
$$

The acceleration profiles along $\mathrm{x}$-axis and $\mathrm{y}$-axis of the final trajectory are presented as

$$
\begin{aligned}
& A_{x}=2 P_{x} \cdot \frac{1}{t_{f}^{2}} \\
& A_{y}=\left\{\begin{array}{l}
4 \cdot W_{\text {lane }} \cdot \frac{1}{t_{f}^{2}},\left(0 \leq T_{M D C A S} \leq \frac{1}{2} t_{f}\right) \\
-4 \cdot W_{\text {lane }} \cdot \frac{1}{t_{f}^{2}},\left(\frac{1}{2} t_{f} \leq T_{M D C A S} \leq t_{f}\right)
\end{array}\right.
\end{aligned}
$$

Moreover, the velocity profiles of the ego vehicle on the global coordinate along $\mathrm{x}$-axis and $\mathrm{y}$-axis can be represented as

$$
\begin{aligned}
& V_{x}=V_{x o}+A_{x} \cdot t_{f} \\
& V_{y}=V_{y o}+A_{y} \cdot t_{f}
\end{aligned}
$$

Optimal rapidly-exploring random tree algorithm is a motion planning algorithm for a vehicle travels through a known costmap [14], which was applied to find the safest trajectory on the global coordinate based on the existing POM, given starting point and endpoint of the path. Some vehicle constraints are also implemented with customized values in RRT* such as tolerance around goal pose, the connection between consecutive poses, and the minimum turning radius of the vehicle. Meanwhile, a path smoother is used to smooth the path utilizing the cubic spline interpolation, which will generate the desired yaw angle along the safest trajectory.

\section{Simulation Results}

To verify the proposed multi-directional collision avoidance system, some simulation results on two examples of potential "side collision" and "rear-end collision" are shown in Fig. 5 to Fig. 12. In "side collision" scenario, the ego vehicle drives at a speed of $23 \mathrm{~m} / \mathrm{s}$, vehicle 2 is in front of the ego vehicle with a lower speed of $20 \mathrm{~m} / \mathrm{s}$. Vehicle 4 is located at the back of the ego vehicle with velocity traveling speed of $23 \mathrm{~m} / \mathrm{s}$, and vehicle 3 is at the rear-right side of the 
ego vehicle with higher longitudinal speed of $26 \mathrm{~m} / \mathrm{s}$ and lateral speed of $0.95 \mathrm{~m} / \mathrm{s}$ to left. Thus, the ego vehicle risks from front-end and rear-right side have been increased, which are also presented as shadow areas on POM in Fig. 5. In order to prevent potential crashes, the risk assessment of trajectory candidates based on the previous determination procedures is also in Fig. 6. From the data analysis,

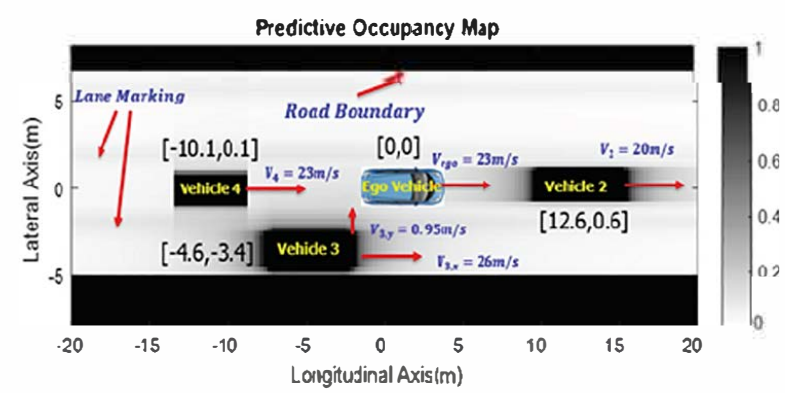

Fig. 5. Vehicle side collision scenario on POM.

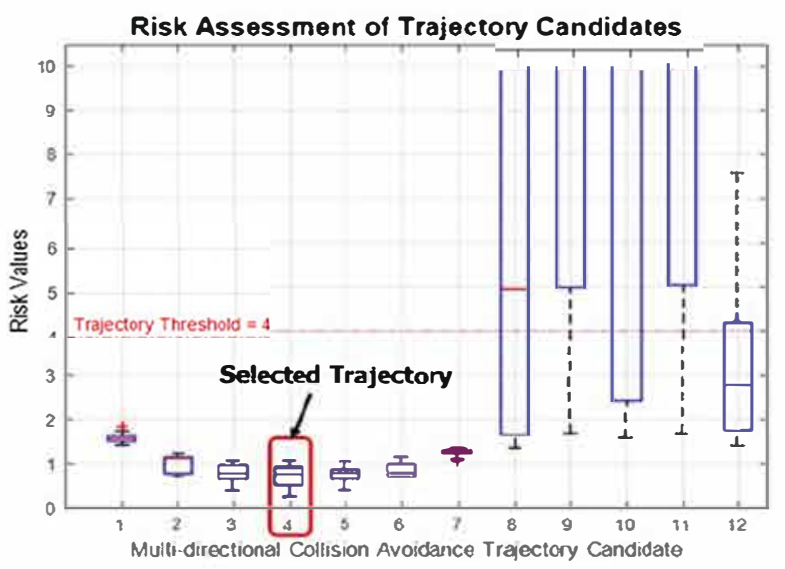

Fig. 6. Risk assessment among 12 trajectory candidates.



Fig. 7. Selected safest trajectory (shown as the path in red).
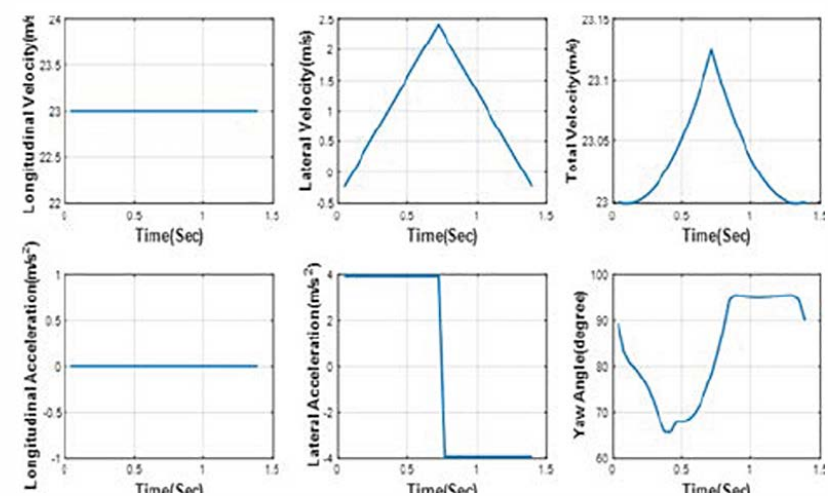

Fig. 8. Planned motion profiles when MDCAS is activated. trajectory 4 was selected with a left lane change of the ego vehicle, which has the lowest risk. After the accelerationbased path is planned, the vehicle's collision avoidance movement path in a global coordinate is also generated using RRT* (shown in Fig. 7). The red trajectory is the desired path of the ego vehicle, which has the lowest risk. In the planned vehicle motion profiles, the ego vehicle longitudinal speed is maintained while its lateral acceleration is increased. As can be observed in Fig. 8, the lateral acceleration to left is generated, and then the lateral acceleration to the opposite direction is applied once arriving at the middle point of the lane to make the vehicle lateral speed of zero. The desired vehicle yaw angle along the optimum path is also generated. A 90-degree yaw angle means that the ego vehicle is driving from left side to right side along the path in Fig.7.

In the "rear-end collision" scenario, the ego vehicle drives at a speed of $23 \mathrm{~m} / \mathrm{s}$, vehicle 2 is in front of the ego vehicle with a much lower speed of $13 \mathrm{~m} / \mathrm{s}$. Vehicle 4 is located at the back of the ego vehicle with velocity traveling speed of $24 \mathrm{~m} / \mathrm{s}$, and vehicle 3 is at the rear-right side of the ego vehicle with higher longitudinal speed of $26 \mathrm{~m} / \mathrm{s}$. Thus, the ego vehicle risks from front-end and rear-end side have been increased, which are also presented as shadow areas on POM in Fig. 9. In order to prevent potential crashes, the risk assessment of trajectory candidates based on the previous detemination procedures is also in Fig. 10. From the data analysis, trajectory 4 of a left lane change was selected with the lowest risk. After the acceleration-profile is determined, the global collision-free trajectory was



Fig. 9. Vehicle rear-end collision scenario on POM.

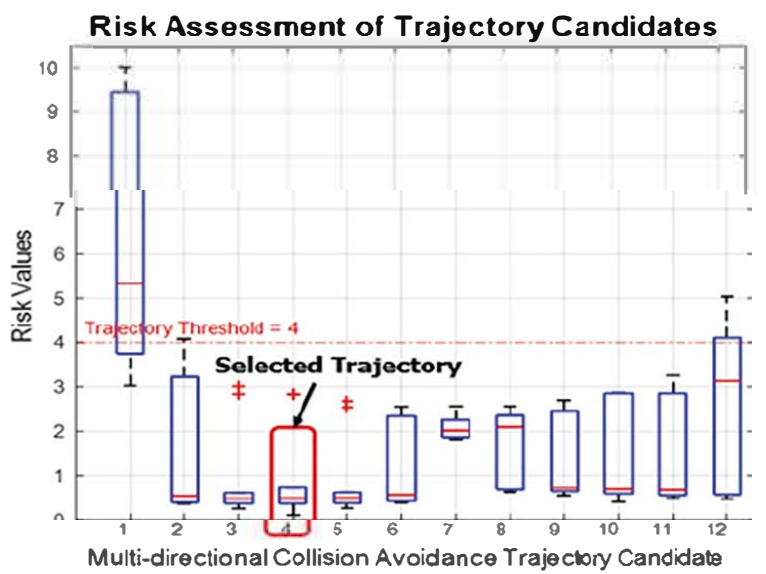

Fig. 10. Risk assessment among 12 trajectory candidates. 
obtained as well as the planned motion profiles in Fig. 11 and Fig. 12, respectively.

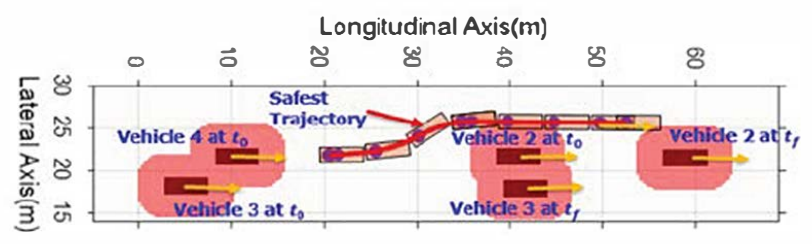

Fig. 11. Selected safest rajectory (shown as the path in red).
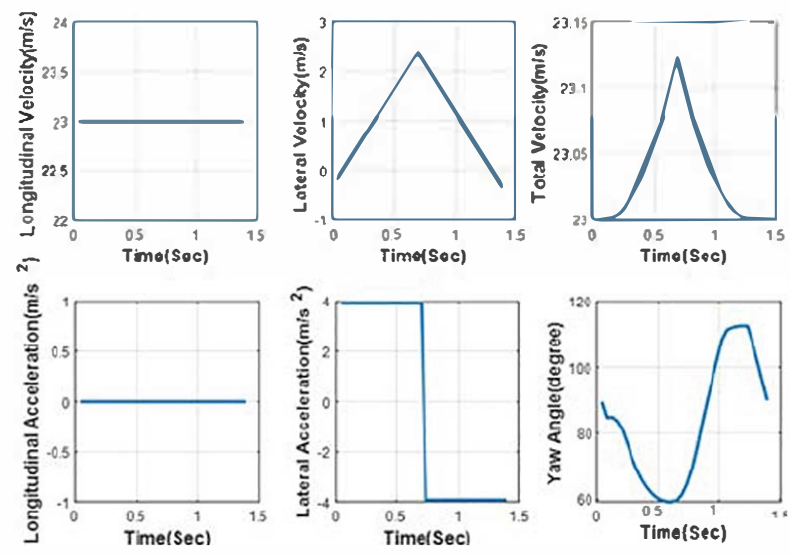

Fig. 12. Planned motion profiles when MDCAS activated.

\section{CONCLUSIONS}

This paper presented the development of a multidirectional collision avoidance system using the constant acceleration vehicle prediction model based on the predictive occupancy map. It has been shown that the proposed CAS is able to avoid the potential crashes from all directions of the ego vehicle. Both vehicle moving information and the static road environment were modeled and represented using the spatial and temporal space of POM with the visualization of risks. Trajectory candidates were pre-defined and utilized to obtain the safest crash mitigation path by comparing multiple trajectories. These acceleration-based trajectory candidates were generated according to the vehicle acceleration limit, which will maintain vehicle stability when CAS is activated. Two representative "side collision" and "rear-end collision" scenarios were applied to verify that the proposed algorithm can execute successfully to avoid potential lateral and rear-end crashes. The simulation results demonstrated the effectiveness and applicability of the proposed system.

\section{REFERENCES.}

[1] Ojsteršek, Tina Cvahte, and Darja Topolšek. "Influence of drivers' visual and cognitive attention on their perception of changes in the traffic environment." European Transport Research Review 11, no. 1 (2019): 1-9.

[2] D. Sam, C. Velanganni, and T. E. Evangelin, "A vehicle control system using a time synchronized hybrid VANET to reduce road accidents caused by human error," Veh. Commun., vol. 6, pp.17_28, Oct. 2016.

[3] R. Tian, K. Ruan, L. Li, J. Le, J. Greenberg, and S. Barbat, "Standardized evaluation of camera-based driver state monitoring systems," IEEE/CAA Journal of Automatica Sinica, vol. 6, no. 3, pp. 716-732, May 2019.

[4] F. Favarò, S. Eurich, and N. Nader, "Autonomous vehicles' disengagements: Trends, triggers, and regulatory limitations," Accident Anal. Prevention, vol. 110, pp. 136148, Jan. 2018.

[5] Yan, Yongjun, Jinxiang Wang, Kuoran Zhang, Mingcong Cao, and Jiansong Chen. "Path Planning using a Kinematic Driver-Vehicle-Road Model with Consideration of Driver's Characteristics." In 2019 IEEE Intelligent Vehicles Symposium (IV), pp. 2259-2264. IEEE, 2019.

[6] J. Kim and D. Kum, "Collision risk assessment algorithm via lane-based probabilistic motion prediction of surrounding vehicles," IEEE Trans. Intell. Transp. Syst., vol. 19, no. 9, pp. 2965-2976, Sep. 2018.

[7] B. Kim and K. Yi, "Probabilistic and holistic prediction of vehicle states using sensor fusion for application to integrated vehicle safety systems," IEEE Trans. Intell. Transp. Syst., vol. 15, no. 5, pp. 2178-2190, May 2014.

[8] Huang, Yanjun, Haitao Ding, Yubiao Zhang, Hong Wang, Dongpu Cao, Nan Xu, and Chuan Hu. "A Motion Planning and Tracking Framework for Autonomous Vehicles Based on Artificial Potential Field-Elaborated Resistance Network (APFE-RN) Approach." IEEE Transactions on Industrial Electronics (2019).

[9] J. Tan, C. Xu, L. Li, F.-Y. Wang, D. Cao, and L. Li, "Guidance control for parallel parking tasks," IEEE/CAA Journal of Automatica Sinica, vol. 7, no. 1, pp. 301-306, January 2020.

[10] X. Zhao, K. Yan, H. Mo, and L. Li, "Type-2 fuzzy control for driving state and behavioral decision of unmanned vehicle," IEEE/CAA Joumal of Automatica Sinica, vol. 7, no. 1, pp. 178-186, January 2020.

[11] B.-L. Ye, W. Wu, K. Ruan, L. Li, T. Chen, H. Gao, and Y. Chen, "A survey of model predictive control methods for traffic signal control," IEEE/CAA Journal of Automatica Sinica, vol. 6, no. 3, pp. 623-640, May 2019.

[12] Lee, Kibeom, and Dongsuk Kum. "Collision Avoidance/Mitigation System: Motion Planning of Autonomous Vehicle via Predictive Occupancy Map." IEEE Access 7 (2019): 52846-52857.

[13] W. F. Milliken and D. L. Milliken, Race CarVehicle Dynamics. Pittsburgh, PA, USA: SAE International, 1995, pp. 345-359.

[14] Liu, Ben, Wenzhao Feng, Tingting $\mathrm{Li}$, Chunhe $\mathrm{Hu}$, and Junguo Zhang. "A Variable-step RRT* Path Planning Algorithm for Quadrotors in Below--canopy." IEEE Access (2020). 